\title{
THE SYMPLECTIC IDEAL AND A DOUBLE CENTRALISER THEOREM
}

\author{
RUDOLF TANGE
}

\begin{abstract}
Summary. We interpret a result of S. Oehms as a statement about the symplectic ideal. We use this result to prove a double centraliser theorem for the symplectic group acting on $\bigoplus_{r=0}^{s} \otimes^{r} V$, where $V$ is the natural module for the symplectic group. This result was obtained in characteristic zero by H. Weyl. Furthermore we use this to extend to arbitrary connected reductive groups $G$ with simply connected derived group the earlier result of the author that the algebra $K[G]^{\mathfrak{g}}$ of infinitesimal invariants in the algebra of regular functions on $G$ is a unique factorisation domain.
\end{abstract}

\section{INTRODUCTION}

Throughout this note $k$ denotes an infinite field, $K$ denotes the algebraic closure of $k, n$ and $s$ are positive integers, $V$ is the vector space $k^{n}$ and Mat $_{n}=\operatorname{Mat}_{n}(k)$ is the $k$-algebra of $n \times n$-matrices acting on $V$ as vector space endomorphisms. We denote the vector space $\bigoplus_{r=0}^{s} \otimes^{r} V$ by $T^{\leq s}(V)$. A matrix $u \in$ Mat $_{n}$ acts on $\otimes^{s} V$ by $u\left(x_{1} \otimes \cdots \otimes x_{s}\right)=u\left(x_{1}\right) \otimes \cdots \otimes u\left(x_{s}\right)$. For a subset $X$ of $\operatorname{Mat}_{n}$ we denote by $\mathcal{E}^{s}(X)$ and $\mathcal{E}^{\leq s}(X)$ the enveloping algebra of $X$ in $\operatorname{End}_{k}\left(\otimes^{s} V\right)$ and $\operatorname{End}_{k}\left(T^{\leq s}(V)\right)$ respectively, that is, the subalgebra generated by the endomorphisms corresponding to the elements of $X$. Let $A$ be an associative $k$-algebra. The centraliser algebra ${ }^{1}$ of a subalgebra $B$ of $A$ is defined as the subalgebra of $A$ that consists of the elements of $A$ that commute with all elements of $B$. We say that the double centraliser theorem holds for a subalgebra $B$ of $A$ if $B$ is equal to the centraliser algebra of its centraliser algebra. We say that the double centraliser theorem holds for a subset $X$ of Mat ${ }_{n}$ acting on $\otimes^{s} V$ or $T^{\leq s}(V)$ if it holds for the corresponding enveloping algebra.

Now assume that $k=K=\mathbb{C}$ and let $G$ be a subgroup of $\mathrm{GL}_{n}(\mathbb{C})$. In [3] Brauer showed that, if the double centraliser theorem holds for $G$, one can determine defining equations for $\mathcal{E}^{s}(G)$ by first determining the centraliser algebra. Determining the centraliser algebra turned out to be equivalent to a version of the first fundamental theorem of invariant theory for $G .^{2}$ His methods applied to any semisimple complex Lie subgroup $G$ of $\mathrm{GL}_{n}(\mathbb{C})$ for which there is a first fundamental theorem of invariant theory, in particular to the symplectic group

\footnotetext{
Key words and phrases. symplectic ideal, double centraliser theorem, infinitesimal invariants.

2000 Mathematics Subject Classification. 14L35, 20G15.

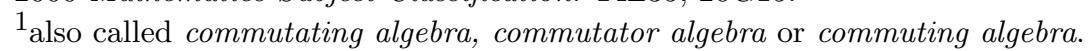

${ }^{2}$ In the case $G=\mathrm{GL}_{n}$ the centraliser algebra was already known and therefore yielded another proof of this version of the first fundamental theorem for $\mathrm{GL}_{n}$. In characteristic zero one can deduce from this the general first fundamental theorem for $\mathrm{GL}_{n}$. See [20] p.139.
} 
$\mathrm{Sp}_{2 m}(\mathbb{C})$. The point is that in these cases all finite dimensional Lie group representations of $G$ are semisimple and the double centraliser theorem holds for semisimple subalgebras of $\operatorname{Mat}_{n}(\mathbb{C})$.

Let $I$ be the ideal of polynomial functions on $\mathrm{Mat}_{2 m}$ that vanish on $\mathrm{Sp}_{2 m}$. In [20] Weyl showed that finding generators $g_{1}, \ldots, g_{r}$ of $I$ which have the property that any $f \in I$ can be expressed as $f=\sum_{i} a_{i} g_{i}$ with $\operatorname{deg}\left(a_{i} g_{i}\right) \leq$ $\operatorname{deg}(f)$ for certain polynomial functions $a_{1}, \ldots, a_{r}$ on $\mathrm{Mat}_{2 m}$, is equivalent to determining defining equations for $\mathcal{E}^{\leq s}\left(\mathrm{Sp}_{2 m}\right)$, for $s$ arbitrary. Then he used Brauer's method, which also applies to $T^{\leq s}(V)$, to determine such generators of $I$.

For the classical groups the first fundamental theorem of invariant theory has been generalised to positive characteristic by De Concini and Procesi in [6], but the algebras $\mathcal{E}^{s}(G)$ and $\mathcal{E}^{\leq s}(G), G$ classical, are no longer semisimple for all $s$ in positive characteristic. In Sections 1 and 3 of this note we will reverse Weyl's procedure and show that we can easily derive the double centraliser theorem for $\mathrm{Sp}_{2 m}$ acting on $T^{\leq s}(V)$ once we have generators for the symplectic ideal with the aforementioned property. All this relies on work of Oehms and De Concini and Procesi.

In Section 2 we derive another consequence from the results on the symplectic ideal in Section 1. We show that for a connected reductive group $G$ with simply connected derived group, the algebra $K[G]^{\mathfrak{g}}$ of infinitesimal invariants in the algebra of regular functions on $G$ is a unique factorisation domain.

\section{The SYMPLECTIC IDEAL AND The SYMPLECTIC ENVELOPING ALGEBRA}

We begin by considering the following property of a set of generators $g_{1}, \ldots, g_{r}$ of an ideal $I$ in the polynomial ring $k\left[x_{1}, \ldots, x_{n}\right]$ :

Any $f \in I$ can be expressed as

$$
f=\sum_{i=1}^{r} a_{i} g_{i} \text { with } \operatorname{deg}\left(a_{i} g_{i}\right) \leq \operatorname{deg}(f)
$$

for certain $a_{1}, \ldots, a_{r} \in k\left[x_{1}, \ldots, x_{n}\right]$.

This property is related to the associated graded ideal for which we now introduce some notation. Let $A$ be a commutative algebra over $k$ with a filtration $A_{0} \subseteq A_{1} \subseteq A_{2} \cdots$. The associated graded algebra is denoted by $\operatorname{gr}(A)$. If $x \in A_{i} \backslash A_{i-1}$, then we put $\operatorname{deg}(x)=i$ and $\operatorname{gr}(x)=x+A_{i-1} \in \operatorname{gr}(A)^{i}=A_{i} / A_{i-1}$. For an ideal $I$ of $A$ we have $\operatorname{gr}(A / I) \cong \operatorname{gr}(A) / \operatorname{gr}(I)$; see [2], Chapter $3, \S 2.4$. In particular, when $A$ is graded, we have $\operatorname{gr}(A / I) \cong A / \operatorname{gr}(I)$.

Lemma 1. Let I be an ideal of the polynomial ring $k\left[x_{1}, \ldots, x_{n}\right]$ which is generated by the nonzero elements $g_{1}, \ldots, g_{r}$. Then these generators have property (1) if and only if the elements $\operatorname{gr}\left(g_{1}\right), \ldots, \operatorname{gr}\left(g_{r}\right)$ generate the ideal $\operatorname{gr}(I)$.

Proof. Assume that $g_{1}, \ldots, g_{r}$ is set of generators of $I$ which have property (1) and let $\operatorname{gr}(f)$ be a nonzero homogeneous element of $\operatorname{gr}(I)$. Then $f=\sum_{i=1}^{r} a_{i} g_{i}$ with $\operatorname{deg}\left(a_{i} g_{i}\right) \leq \operatorname{deg}(f)$ for certain $a_{1}, \ldots, a_{r} \in k\left[x_{1}, \ldots, x_{n}\right]$. So $\operatorname{gr}(f)=$ $\sum_{i} \operatorname{gr}\left(a_{i}\right) \operatorname{gr}\left(g_{i}\right)$ where the sum is over all indices $i$ such that $\operatorname{deg}\left(a_{i} g_{i}\right)=\operatorname{deg}(f)$. Thus the $\operatorname{gr}\left(g_{i}\right)$ generate $\operatorname{gr}(I)$. Now assume that the latter is the case and let 
$f$ be a nonzero element of $I$. We can write $\operatorname{gr}(f)=\sum_{i=1}^{r} a_{i} \operatorname{gr}\left(g_{i}\right)$ where $a_{i}$ is homogeneous of degree $\operatorname{deg}(f)-\operatorname{deg}\left(g_{i}\right)$. Then $f-\sum_{i=1}^{r} a_{i} g_{i}$ is of strictly lower degree than $f$ and we can finish by induction.

The symmetric group of degree $r$, $\operatorname{Sym}_{r}$, acts on $\otimes^{r} V$ via $\pi \cdot\left(x_{1} \otimes \cdots \otimes x_{r}\right)=$ $x_{\left(\pi^{-1}\right)_{1}} \otimes \cdots \otimes x_{\left(\pi^{-1}\right)_{r}}$. Denote the group algebra of $\operatorname{Sym}_{r}$ over $k$ by $k\left\langle\operatorname{Sym}_{r}\right\rangle$. The elements of $\operatorname{End}_{k\left\langle\operatorname{Sym}_{r}\right\rangle}\left(\otimes^{r} V\right)$, i.e. the $k$-linear endomorphisms of $\otimes^{r} V$ commuting with the action of $\mathrm{Sym}_{r}$, were called bisymmetric substitutions by Weyl. Clearly all enveloping algebras $\mathcal{E}^{r}(X)$ consist of bisymmetric substitutions.

To state the proposition below we need some auxiliary notation. For a subset $S$ of a fixed finite dimensional $k$-vector space $W$ we denote the $k$-algebra of polynomial functions on $S$, i.e. functions on $S$ that are restrictions of polynomial functions on $W$, by $k[S]$. The algebra $k[W]$ is a polynomial algebra in the elements of any basis of $W^{*}$, because $k$ is infinite. In particular, $k\left[\mathrm{Mat}_{n}\right]$ is a polynomial algebra in the matrix entry functions.

Now take $W=$ Mat $_{n}$. Clearly $k[S]$ is isomorphic to $k\left[\mathrm{Mat}_{n}\right] / I$, where $I$ is the ideal of polynomial functions on $\mathrm{Mat}_{n}$ that vanish on $S$. The algebra $k[S]$ inherits a filtration from $k\left[\mathrm{Mat}_{n}\right]$. We denote the filtration subspace of index $s$ by $k[S]^{\leq s}$. If $S$ is closed under multiplication by nonzero scalars, then the ideal $I$ will be homogeneous and $k[S]$ is a graded algebra. We denote the graded subspace of index $s$ by $k[S]^{s}$. If $S$ is a submonoid of $\mathrm{Mat}_{n}$, then $I$ is a biideal and $k[S]$ is a $k$-bialgebra. The subspace $k[S]^{\leq s}$ is then a subcoalgebra of $k[S]$ and if $S$ contains the nonzero multiples of the identity, then $k[S]^{s}$ is a subcoalgebra of $k[S]$. We denote the closure of a subset $S$ of Mat ${ }_{n}$ under multiplication by nonzero scalars by $k^{\times} S$. Note that in this notation $k\left[\mathrm{GL}_{n}\right]$ is equal to $k\left[\mathrm{Mat}_{n}\right]$ and not to $k\left[\mathrm{Mat}_{n}\right]\left[\operatorname{det}^{-1}\right]$.

After Proposition 1 we will only use this notation in the following situation where it is in accordance with the standard notation. If $X$ is a $k$-defined closed subvariety of $W(K):=K \otimes_{k} W$ such that the set $S$ of $k$-defined points in $X$ is dense in $X$, then $k[S]$ in the notation here is naturally isomorphic to the algebra $k[X]$ of regular functions on $X$ that are defined over $k$. This applies for example to the symplectic group $\mathrm{Sp}_{n}$ as we shall see later. The next proposition is essentially due to Schur and Weyl.

Proposition 1. Let $M$ be a submonoid of $\mathrm{Mat}_{n}$. Then

(i) the natural map $\mathcal{E}^{s}(M)^{*} \rightarrow k\left[k^{\times} M\right]^{s}$ is an isomorphism of coalgebras.

(ii) the natural map $\mathcal{E}^{\leq s}(M)^{*} \rightarrow k[M]^{\leq s}$ is an isomorphism of coalgebras.

(iii) $\mathcal{E}^{s}\left(\mathrm{GL}_{n}\right)=\operatorname{End}_{k\left\langle\operatorname{Sym}_{s}\right\rangle}\left(\otimes^{s} V\right)$.

(iv) $\mathcal{E}^{\leq s}\left(\mathrm{GL}_{n}\right)=\operatorname{End}_{\mathfrak{S}}(T \leq s(V))$, where $\mathfrak{S}=\bigoplus_{r=0}^{s} k\left\langle\operatorname{Sym}_{r}\right\rangle$.

Proof. The natural map in (ii) composes $f \in \mathcal{E}^{\leq s}(M)^{*}$ with the monoid homomorphism $M \rightarrow \mathcal{E}^{\leq s}(M)$. The result is clearly a polynomial function on $M$ of degree $\leq s$. That the natural map is injective follows from the fact that endomorphisms of $T^{\leq s}(V)$ that represent the elements of $M$ span $\mathcal{E}^{\leq s}(M)$, since $M$ is a submonoid of $\mathrm{Mat}_{n}$.

The algebra $\mathcal{E}^{s}(M)$ is equal to $\mathcal{E}^{s}\left(k^{\times} M\right)$, since $\alpha \cdot$ id $\in$ Mat $_{n}$ is represented by $\alpha^{s}$.id on $\otimes^{s} V$. So in the case of (i) we may assume that $M$ contains the nonzero 
multiples of the identity. The injectivity of the natural map, which composes $f \in \mathcal{E}^{s}(M)^{*}$ with $M \rightarrow \mathcal{E}^{s}(M)$, now follows as in (ii).

By considering the commutative diagram below and its graded version in case $M$ contains the multiples of the identity, we see that it is now sufficient to prove (i) and (ii) for $M=\mathrm{Mat}_{n}$, since the two vertical arrows are surjective morphisms of coalgebras.

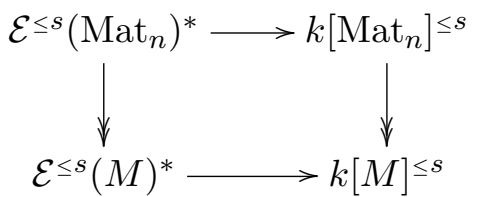

We first consider the natural map of (i) for $M=\mathrm{GL}_{n}$. To avoid confusion, we will write $k\left[\mathrm{Mat}_{n}\right]$ instead of $k\left[\mathrm{GL}_{n}\right]$, since these algebras are canonically isomorphic. The map comes from the natural map $\operatorname{End}_{k}\left(\otimes^{s} V\right)^{*} \rightarrow k\left[\mathrm{Mat}_{n}\right]^{s}$. Let $I=I(n, s)=\{1, \ldots, n\}^{s}$ as in $[12,2.1]$. We have a standard basis $\left(E_{i, j}\right)_{(i, j) \in I \times I}$ of $\operatorname{End}_{k}\left(\otimes^{s} V\right)$. Our map maps the element $E_{i, j}^{*}$ of the dual basis to Green's $c_{i, j}$. The $c_{i, j}$ form a basis when we pass to $(I \times I) / \sim$, where $\sim$ is the equivalence relation given by the action of the $\operatorname{Sym}_{s}$ on $I \times I$ defined in [12, 2.1]. Now we form the dual basis $c_{(i, j)}^{*}$ and define $c_{i, j}^{*}=c_{(i, j)}^{*}$. Here $\overline{(i, j)}$ denotes the canonical image of $(i, j)$ in $(I \times I) / \sim$. Then the transpose of our map maps $c_{i, j}^{*}$ to $\sum_{(k, l) \sim(i, j)} E_{k, l}$, which is precisely what Green's map $\left(k\left[\operatorname{Mat}_{n}\right]^{s}\right)^{*} \rightarrow \operatorname{End}_{k}\left(\otimes^{s} V\right)$ does. Assertion (i) for $\mathrm{GL}_{n}$ and (iii) now follow from $[12,2.6]$. Note that (iii) shows that $\mathcal{E}^{s}\left(\mathrm{Mat}_{n}\right)=\mathcal{E}^{s}\left(\mathrm{GL}_{n}\right)$.

Now we consider the natural map of (ii) for $M=\mathrm{GL}_{n}$. We have seen that it is injective. It is also surjective, since for any polynomial function $f$ on $\mathrm{GL}_{n}$ of degree $\leq s$ we can find an element of $\operatorname{End}\left(T^{\leq s}(V)\right)^{*} \cong T^{\leq s}\left(\mathrm{Mat}_{n}^{*}\right)$ that is mapped to $f$. We have $\mathcal{E}^{\leq s}\left(\mathrm{GL}_{n}\right) \subseteq \bigoplus_{r=0}^{s} \mathcal{E}^{r}\left(\mathrm{GL}_{n}\right)$. Since the natural maps of (i) and (ii) for $M=\mathrm{GL}_{n}$ are bijective we must have equality. It is now also clear that (ii) is an isomorphism of coalgebras.

Since the image of $\mathfrak{S}$ in $\operatorname{End}_{k}\left(T^{\leq s}(V)\right)$ contains the projections given by the direct sum decomposition of $T^{\leq s}(V)$, we have that $\operatorname{End}_{\mathfrak{S}}\left(T^{\leq s}(V)\right)$ is equal to $\bigoplus_{r=0}^{s} \operatorname{End}_{k\left\langle\operatorname{Sym}_{r}\right\rangle}\left(\otimes^{r} V\right)$ : it consists of $(s+1)$-tuples of "bisymmetric substitutions". So (iv) now follows from (iii) and it is now also clear that $\mathcal{E}^{\leq s}\left(\mathrm{GL}_{n}\right)=$ $\mathcal{E} \leq s\left(\mathrm{Mat}_{n}\right)$.

Remarks 1. 1. Assertion (iv) of Proposition 1 was proved in a different way by Weyl, see [20, Thm. 4.4.E].

2 . Proposition 1 shows that $\mathcal{E}^{\leq s}(M)$ only depends on the ideal $I$ of polynomial functions that vanish on $M$ and that $\mathcal{E}^{s}(M)$ only depends on the largest homogeneous ideal contained in $I$, that is, the ideal generated by the homogeneous polynomial functions on $\mathrm{Mat}_{n}$ that vanish on $M$.

3. Assume that $k=K$ is algebraically closed. Let $G$ be a closed subgroup of $\mathrm{GL}_{n}$ and let $A$ be the algebra of polynomial functions on $G$. Then the representations of the algebra $\mathcal{E}^{\leq s}(G)=\left(A^{\leq s}\right)^{*}$ are precisely the rational representations of $G$ whose coefficients are polynomial functions on $G$ that are of filtration degree $\leq s$. If $G$ contains the nonzero multiples of the identity, then 
the representations of the algebra $\mathcal{E}^{S}(G)=\left(A^{s}\right)^{*}$ are precisely the rational representations of $G$ whose coefficients are homogeneous polynomial functions on $G$ that are of degree $s$. In case $G=\mathrm{GL}_{n},\left(A^{s}\right)^{*}$ is the well-known Schur algebra denoted by $S_{K}(n, s)$ in [12]. Similar remarks apply to a closed submonoid $M$ of Mat $_{n}$. Then the coefficients of a rational representation of $M$ are automatically polynomial, since all regular functions on $M$ are polynomial.

We now come to the problem of finding defining equations for the enveloping algebras $\mathcal{E}^{s}(M)$ and $\mathcal{E}^{\leq s}(M)$. We are only interested in defining equations within $\mathcal{E}^{s}\left(\right.$ Mat $\left._{n}\right)$ and $\mathcal{E}^{\leq s}\left(\right.$ Mat $\left._{n}\right)$. By Proposition 1(iii) and (iv) one can then obtain a complete set of defining equations (within $\operatorname{End}_{k}\left(\otimes^{s} V\right)$ and $\left.\oplus_{r=0}^{s} \operatorname{End}_{k}\left(\otimes^{r} V\right)\right)$ by adding the equations of "bisymmetry". See [3, (30)] or [20, III (6.8)]. Statement (i) of the next corollary is a formalisation of an idea of Weyl; see [20, p 142].

Corollary. Let $M$ be a submonoid of $\mathrm{Mat}_{n}$, let $I$ be the ideal of polynomial functions on Mat $_{n}$ that vanish on $M$ and let $I_{\text {hom }}$ be the largest homogeneous ideal contained in $I$. Furthermore, let $g_{1}, \ldots, g_{k}$ be nonzero elements of I and let $h_{1}, \ldots, h_{l}$ be nonzero homogeneous elements of $I_{\text {hom }}$. Denote the isomorphism $k\left[\mathrm{Mat}_{n}\right]^{\leq s} \rightarrow \mathcal{E} \leq s\left(\mathrm{Mat}_{n}\right)^{*}$ by $\eta$ and the isomorphism $k\left[\mathrm{Mat}_{n}\right]^{s} \rightarrow \mathcal{E}^{s}\left(\mathrm{Mat}_{n}\right)^{*}$ by $\theta$. Then

(i) The elements $g_{1}, \ldots, g_{k}$ are generators of I with property (1) if and only if for each $s \geq 1$ the functionals $\eta\left(g_{i} m_{i}\right)$, where the $m_{i}$ are arbitrary monomials in the matrix entries of degree $\leq s-\operatorname{deg}\left(g_{i}\right)$, define the algebra $\mathcal{E}^{\leq s}(M)$.

(ii) The elements $h_{1}, \ldots, h_{k}$ are generators of $I_{h o m}$ if and only if for each $s \geq 1$ the functionals $\theta\left(h_{i} m_{i}\right)$, where the $m_{i}$ are arbitrary monomials in the matrix entries of degree $s-\operatorname{deg}\left(h_{i}\right)$, define the algebra $\mathcal{E}^{s}(M)$.

Proof. Note that $I$ is a proper ideal, since $M$ is nonempty. Denote the subspace of $I$ that consists of the elements of $I$ of degree $\leq s$ by $I^{\leq s}$. Then the $g_{i}$ are generators of $I$ with property (1) if and only if for each $s \geq 1$ the polynomials $g_{i} m_{i}$, where the $m_{i}$ are arbitrary monomials in the matrix entries of degree $\leq s-\operatorname{deg}\left(g_{i}\right)$, span $I^{\leq s}$. Assertion (i) now follows from the fact that, because of the commutativity of (2), $\eta$ maps $I^{\leq s}$ to the space of linear functionals on $\mathcal{E}^{\leq s}\left(\mathrm{Mat}_{n}\right)$ that vanish on $\mathcal{E}^{\leq s}(M)$. Assertion (ii) is proved in a similar way.

Remark. In [9, Thm.'s 9.5a) and 10.5a)] Doty determines generators for the defining ideals of the symplectic and orthogonal monoids in characteristic zero by a method which is essentially assertion (ii) of the above corollary. The point is that in characteristic zero one can use the defining equations of the symplectic and orthogonal enveloping algebras in $\operatorname{End}_{k}\left(\otimes^{s} V\right)$ as obtained by Brauer in [3, $(46),(47)]$

In the remainder of this note $n=2 m$ is an even integer $\geq 2$. We first introduce some notation for the symplectic group which closely follows that of [15]. Let $i \mapsto i^{\prime}$ be the involution of $\{1, \ldots, n\}$ defined by $i^{\prime}:=n+1-i$. Set $\epsilon_{i}=1$ if $i \leq m$ and $\epsilon_{i}=-1$ if $i>m$ and define the $n \times n$-matrix $J$ with coefficients in $k$ by $J_{i j}=\delta_{i j^{\prime}} \epsilon_{i}$. So 


$$
J=\left[\begin{array}{rrrrrr} 
& & & & & \\
& 0 & & & . \cdot & \\
& & & -1 & & \\
& . & & & 0 \\
-1 & & & &
\end{array}\right] .
$$

On $V$ we define the nondegenerate symplectic form $\langle$,$\rangle by$

$$
\langle u, v\rangle:=u^{T} J v=\sum_{i=1}^{n} \epsilon_{i} u_{i} v_{i^{\prime}} .
$$

The symplectic group $\operatorname{Sp}_{n}=\operatorname{Sp}_{n}(k)$ is defined as the set of $n \times n$-matrices over $k$ that satisfy $A^{T} J A=J$, i.e. the matrices for which the corresponding endomorphism of $V$ preserves the form $\langle$,$\rangle . Clearly those matrices are invertible and$ $\mathrm{Sp}_{n}$ is a subgroup of $\mathrm{GL}_{n}$. Furthermore $\mathrm{Sp}_{n}(K)$ is an algebraic group which is connected, semisimple and defined and split over the prime field. This implies that all root subgroups $U_{\alpha}(K)$ with respect to some $k$-split maximal torus are defined over $k$. Since clearly $U_{\alpha}(k)$ is dense in $U_{\alpha}(K)$, we must have that $\operatorname{Sp}_{n}$ is dense in $\operatorname{Sp}_{n}(K)$.

Note that $A^{T} J A$ consists of the scalar products of the columns of $A$ with each other and that $A J A^{T}$ consists of the scalar products of the rows of $A$ with each other. An easy calculation shows that the condition $A^{T} J A=J$ is equivalent to the condition $A J A^{T}=J$. We denote the $(i, j)^{\text {th }}$ entry function on $\mathrm{Mat}_{n}$ by $x_{i j}$. Define

$$
g_{i j}:=\sum_{l=1}^{n} \epsilon_{l} x_{l i} x_{l^{\prime} j} \quad \text { and } \quad \bar{g}_{i j}:=\sum_{l=1}^{n} \epsilon_{l} x_{i l} x_{j l^{\prime}}
$$

The condition $A^{T} J A=J$ means that we require the functions $g_{i j}-\delta_{i j^{\prime}} \epsilon_{i}, i<j$, to vanish on $A$. The condition $A J A^{T}=J$ means that we require the functions $\bar{g}_{i j}-\delta_{i j^{\prime}} \epsilon_{i}, i<j$, to vanish on $A$. We call the ideal of polynomial functions on $\mathrm{Mat}_{n}$ that vanish on $\mathrm{Sp}_{n}$ the symplectic ideal. It is well known that each set of functions separately generates the symplectic ideal in case $k$ is algebraically closed. In view of the density of $\mathrm{Sp}_{n}$ in $\mathrm{Sp}_{n}(K)$ this must then also hold for an arbitrary infinite field $k$.

In the sequel we will also need the symplectic monoid $\mathrm{SpM}_{n}$ and the symplectic similitude group $\mathrm{GSp}_{n}$ as introduced in [7] and [9]; see also [15] and [10]. The symplectic monoid $\mathrm{SpM}_{n}$ is defined as the set of matrices $A$ for which there exists a scalar $d(A) \in k$ such that $A^{T} J A=A J A^{T}=d(A) J$. Note that if $d(A) \neq 0, A^{T} J A=d(A) J$ is equivalent to $A J A^{T}=d(A) J$. Clearly $\mathrm{SpM}_{n}$ is a submonoid of $\mathrm{Mat}_{n}$, in fact it is the set of $k$-defined points of $\operatorname{SpM}_{n}(K)$ which is a $k$-defined closed submonoid of $\operatorname{Mat}_{n}(K)$. This follows from [15, Cor. 6.2]. The function $d$ is a polynomial function on $\mathrm{SpM}_{n}$, it is called the coefficient of dilation. We have for all $i \in\{1, \ldots, n\}$

$$
d=\epsilon_{i} g_{i i^{\prime}}=\epsilon_{i} \bar{g}_{i i^{\prime}} \text { on } \mathrm{SpM}_{n} .
$$


The symplectic similitude group $\mathrm{GSp}_{n}$ is defined as the group of invertible elements in $\mathrm{SpM}_{n}$. We have $\mathrm{GSp}_{n}(K)=K^{\times} \mathrm{Sp}_{n}(K)$ and $\mathrm{GSp}_{n}$ is Zariski dense in $\mathrm{GSp}_{n}(K)$ and $\operatorname{SpM}_{n}(K)$. So a polynomial function on Mat ${ }_{n}$ vanishes on $\mathrm{GSp}_{n}$ if and only if it vanishes on $\mathrm{SpM}_{n}$ and the ideal of $k$ [Mat $\left.{ }_{n}\right]$ that consists of these functions is the largest homogeneous ideal contained in the symplectic ideal.

We will now give defining equations for $\mathcal{E}^{\leq s}\left(\mathrm{Sp}_{n}\right)$. As stated before the Corollary to Proposition 1 , we are only interested in defining equations within the algebra $\mathcal{E}^{\leq s}\left(\mathrm{Mat}_{n}\right)$. This algebra consists of $(s+1)$-tuples $\left(A_{0}, \ldots, A_{s}\right)$ of endomorphisms with $A_{r} \in \operatorname{End}_{k}\left(\otimes^{r} V\right)$ bisymmetric for all $r \in\{0, \ldots, s\}$. The standard basis $\left(e_{1}, \ldots, e_{n}\right)$ of $V=k^{n}$ gives a basis for the vector space $\otimes^{r} V$. The entry of index $\left(\left(i_{1}, \ldots, i_{r}\right),\left(j_{1}, \ldots, j_{r}\right)\right)$ of the matrix of $A_{r}$ with respect to this basis is denoted by $a_{i_{1} \cdots i_{r}, j_{1} \cdots j_{r}}$.

The first statement in (i) of the theorem below was already remarked by S. Oehms [15, p 38].

\section{Theorem 1. The following holds}

(i) $\operatorname{gr}(I)$ is generated by the elements $g_{i j}$ and $\bar{g}_{i j}, i<j$. Furthermore, $\operatorname{gr}\left(k\left[\mathrm{Sp}_{n}\right]\right)$ is isomorphic to the algebra of polynomial functions on the set of $n \times n$-matrices over $k$ whose row and column space are totally singular and this algebra is an integral domain.

(ii) The sets of elements $g_{i j}-\delta_{i j^{\prime}} \epsilon_{i}$ and $\bar{g}_{i j}-\delta_{i j^{\prime}} \epsilon_{i}, i<j$, form together a set of generators of the symplectic ideal with property (1).

(iii) The symplectic enveloping algebra in $\operatorname{End}_{k}\left(T^{\leq s}(V)\right)$ is defined by the following equations for $r=2, \ldots, s$

$$
\begin{aligned}
& \sum_{l=1}^{n} \epsilon_{l} a_{l l^{\prime} i_{3} \cdots i_{r}, j_{1} \cdots j_{r}}=\delta_{j_{1}, j_{2}^{\prime}} \epsilon_{j_{1}} a_{i_{3} \cdots i_{r}, j_{3} \cdots j_{r}}, \\
& \sum_{l=1}^{n} \epsilon_{l} a_{i_{1} \cdots i_{r}, l l^{\prime} j_{3} \cdots j_{r}}=\delta_{i_{1}, i_{2}^{\prime}} \epsilon_{i_{1}} a_{i_{3} \cdots i_{r}, j_{3} \cdots j_{r}} .
\end{aligned}
$$

Proof. (i). Let $J$ be the ideal of $k\left[\mathrm{Mat}_{n}\right]$ generated by the elements $g_{i j}$ and $\bar{g}_{i j}$, $i<j$. Then the zero set of $J$ is the set of $n \times n$-matrices over $k$ whose row and column space are totally singular. It is also the zero set of $d$ in $\operatorname{SpM}_{n}$. In fact it follows from $\left[15\right.$, Cor. 6.2] that $k\left[\mathrm{SpM}_{n}\right] /(d) \cong k\left[\mathrm{Mat}_{n}\right] / J$. That result also implies that $k\left[\mathrm{SpM}_{n}\right] /(d-1) \cong k\left[\mathrm{Mat}_{n}\right] / I=k\left[\mathrm{Sp}_{n}\right]$. Since $d$ is nonzero and homogeneous of degree $2>0$, we have $\operatorname{gr}(d-1)=d$ and $\operatorname{gr}((d-1))=(d)$. Therefore

$$
k\left[\mathrm{Mat}_{n}\right] / J \cong k\left[\mathrm{SpM}_{n}\right] /(d) \cong \operatorname{gr}\left(k\left[\mathrm{Sp}_{n}\right]\right) \cong k\left[\mathrm{Mat}_{n}\right] / \operatorname{gr}(I) .
$$

Since the composite is the epimorphism given by the inclusion $J \subseteq \operatorname{gr}(I)$, we must have $J=\operatorname{gr}(I)$.

Let $\mathcal{V}$ be the variety of $n \times n$-matrices over $K$ whose row and column space are totally singular. Then we have an epimorphism $K\left[\mathrm{SpM}_{n}\right] /(d) \rightarrow K[\mathcal{V}]$ of graded $K$-algebras. To show that it is an isomorphism it suffices to show that for each $l$ the dimensions of the graded pieces of degree $l$ of both algebras are equal. By $\left[15\right.$, Thm. 6.1] the $l^{\text {th }}$ graded piece of $K\left[\mathrm{SpM}_{n}\right] /(d)$ has a basis consisting of all bideterminants $\left(T_{1} \mid T_{2}\right)$, where $T_{1}$ and $T_{2}$ are symplectic standard tableaux 
in the sense of King of the same shape $\lambda$ which is a partition of $l$ with at most $m$ parts (notation: $\lambda \vdash_{m} l$ ). So its dimension is $\sum_{\lambda \vdash_{m} l} N_{\lambda}^{2}$, where $N_{\lambda}$ is the number of symplectic standard tableaux in the sense of King of shape $\lambda$. By [5, Thm. 6.1] the $l^{\text {th }}$ graded piece of $K[\mathcal{V}]$ has a basis consisting of all bideterminants $\left(T_{1} \mid T_{2}\right)$, where $T_{1}$ and $T_{2}$ are symplectic standard tableaux in the sense of De Concini of the same shape $\lambda \vdash_{m} l .^{3}$ So its dimension is $\sum_{\lambda \vdash_{m} l} N_{\lambda}^{\prime 2}$, where $N_{\lambda}^{\prime}$ is the number of symplectic standard tableaux in the sense of De Concini of shape $\lambda$. Let $Y(\lambda)$ be the irreducible $\operatorname{Sp}_{n}(\mathbb{C})$-module associated to $\lambda \vdash_{m} l$. Then $N_{\lambda}=\operatorname{dim}(Y(\lambda))=N_{\lambda}^{\prime}$, by [16] (or [1] or [7]) and [5, Thm.'s 4.8, 4.11, Prop. 4.10]. Note that we now also know that $\mathcal{V}$ is defined over $k$.

We will now show that $\mathcal{V}$ is irreducible. Let $\mathcal{V}_{0}$ be the set of $n \times n$-matrices whose row and column space lie in the $K$-span $V_{0}$ of $e_{1}, \ldots, e_{m}$. Note that $V_{0}$ is a maximal totally singular subspace of $V(K)=K^{n}$ and that $\mathcal{V}_{0}$ is a $k$-defined vector subspace of $\operatorname{Mat}_{n}(K)$, where $\operatorname{Mat}_{n}(K)$ has the standard $k$ structure. Now consider the morphism $\operatorname{Sp}_{n}(K) \times \mathcal{V}_{0} \times \operatorname{Sp}_{n}(K) \rightarrow \operatorname{Mat}_{n}(K)$ given by $(A, S, B) \mapsto A S B$. Clearly its image lies in $\mathcal{V}$ and by Witt's Lemma its image is equal to $\mathcal{V}$. Since $\operatorname{Sp}_{n}(K)$ and $\mathcal{V}_{0}$ are irreducible we must have that $\mathcal{V}$ is irreducible. Furthermore the set of $k$-defined points is dense in $\mathcal{V}$, since this holds for $\operatorname{Sp}_{n}(K)$ and $\mathcal{V}_{0}$ and our morphism is defined over $k$. Note that, since $K[\mathcal{V}]$ is an integral domain, $d$ generates a prime ideal in $K\left[\mathrm{SpM}_{n}\right]$ and therefore also in $k\left[\mathrm{SpM}_{n}\right]$.

(ii). This follows from (i) and Lemma 1.

(iii). This follows immediately from (ii) and (i) of the Corollary to Proposition 1.

Remarks 2. 1. The equations in (iii) are the same as those obtained by Weyl $[20, \mathrm{p} 174]$ in characteristic zero.

2. The equations for $\mathcal{E}^{s}\left(\mathrm{Sp}_{n}\right)$ that Brauer [3, (47)] found in characteristic zero also define this algebra in positive characteristic. This can be shown as follows. Let $I$ be the symplectic ideal. Then [15, Cor. 6.2] gives homogeneous generators of $I_{\text {hom }} \cdot{ }^{4}$ Here one can avoid the FRT-construction by simply taking [15, (13)] as the definition of $A_{R}^{s}(n)$. The stability under base change is then trivial and the proofs of [15, Thm 6.1 and Cor. 6.2] still apply. Now (ii) of the Corollary to Proposition 1 gives the following equations for $\mathcal{E}^{s}\left(\mathrm{Sp}_{n}\right)$ within $\mathcal{E}^{s}\left(\mathrm{Mat}_{n}\right)$ :

$$
\delta_{i_{1}, i_{2}^{\prime}} \epsilon_{i_{1}} \sum_{l=1}^{n} \epsilon_{l} a_{l l^{\prime} i_{3} \cdots i_{r}, j_{1} \cdots j_{r}}=\delta_{j_{1}, j_{2}^{\prime}} \epsilon_{j_{1}} \sum_{l=1}^{n} \epsilon_{l} a_{i_{1} \cdots i_{r}, l l^{\prime} j_{3} \cdots j_{r}}
$$

\section{Infinitesimal INVARIANTS IN $K[G]$}

In this section we assume that $k=K$ is algebraically closed. Furthermore, $G$ is a connected reductive algebraic group over $K$ and $\mathfrak{g}=\operatorname{Lie}(G)$ is its Lie algebra. The conjugation action of $G$ on itself induces an action of $G$ on $K[G]$, the algebra of regular functions on $G$. We will refer to this action and its derived

\footnotetext{
${ }^{3}$ De Concini uses different terminology, but it is clear that his condition of symplectic standardness is imposed on $T_{1}$ and $T_{2}$ separately.

${ }^{4}$ In $[15,(14)]$, one can replace $m$ by $n$ and $f_{l l^{\prime}}-f_{k k^{\prime}}$ by $\epsilon_{k} f_{l l^{\prime}}-\epsilon_{l} f_{k k^{\prime}}\left(\right.$ or by $\left.\epsilon_{l} f_{l l^{\prime}}-\epsilon_{k} f_{k k^{\prime}}\right)$.
} 
$\mathfrak{g}$-action as conjugation actions. The conjugation action of $G$ on $K[G]$ is by algebra automorphisms, so the conjugation action of $\mathfrak{g}$ on $K[G]$ is by derivations. This implies that the space $K[G]^{\mathfrak{g}}=\{f \in K[G] \mid x \cdot f=0$ for all $x \in \mathfrak{g}\}$ of infinitesimal invariants is a subalgebra of $K[G]$. Note that $K[G]^{G} \subseteq K[G]^{\mathfrak{g}}$ and that $K[G]^{p}:=\left\{f^{p} \mid f \in K[G]\right\} \subseteq K[G]^{\mathfrak{g}}$ if $K$ is of characteristic $p>0$.

Theorem 2. Assume that $K$ is of characteristic $p>0$ and that the derived group of $G$ is simply connected. Then the invariant algebra $K[G]^{\mathfrak{g}}$ is a unique factorisation domain. Its irreducible elements are the irreducible elements of $K[G]$ that are invariant under $\mathfrak{g}$ and the $p$-th powers of the irreducible elements of $K[G]$ that are not invariant under $\mathfrak{g}$.

Proof. By Remark 3 in [18] we have to prove Proposition 1 in that paper for $\mathrm{Sp}_{n}$. In fact we may assume that $n \geq 4$ and that $K$ is of characteristic 2 , but we will not use this. The statement of Proposition 1 in [18] is:

Let $f \in K[G]$ be a regular function. If the ideal $K[G] f$ is stable under the conjugation action of $\mathfrak{g}$ on $K[G]$, then $f$ is $\mathfrak{g}$-invariant.

By Proposition 2 and Lemma 2 in [18] it is sufficient to show that, for the filtration that $K\left[\mathrm{Sp}_{n}\right]$ inherits from $K\left[\mathrm{Mat}_{n}\right]$, the associated graded algebra is an integral domain. The point is that one can then use a (filtration) degree argument. The result now follows immediately from Theorem 1(ii).

Proposition 2. The algebras $K\left[\mathrm{Sp}_{n}\right], K\left[\mathrm{GSp}_{n}\right]$ and $K\left[\mathrm{SpM}_{n}\right]$ are unique factorisation domains.

Proof. Since the derived group $D \mathrm{GSp}_{n}$ of $\mathrm{GSp}_{n}$ equals $\mathrm{Sp}_{n}$ which is simply connected, we have by $\left[18\right.$, Cor. to Thm. 1] that $K\left[\mathrm{Sp}_{n}\right]$ and $K\left[\mathrm{GSp}_{n}\right]$ are UFD's. We have $K\left[\mathrm{SpM}_{n}\right]\left[d^{-1}\right]=K\left[\mathrm{GSp}_{n}\right]$ which is a UFD. Furthermore $d$ generates a prime ideal in $K\left[\mathrm{SpM}_{n}\right]$ by the proof of Theorem 1(i). So $K\left[\mathrm{SpM}_{n}\right]$ is a UFD by Nagata's Lemma; see e.g. [11, Lemma 19.20].

\section{A Double CENTRALiser theOREM For the SyMPleCtic Group}

We begin by defining a version of the symplectic Brauer algebra. For each integer $r \leq s$ we fix $r$ vector symbols $x_{1}, \ldots, x_{r}$ and $r$ covector symbols $y_{1}, \ldots, y_{r}{ }^{5}$ To ease notation we did not give these symbols the extra index $r$ that indicates to which integer $r \leq s$ they are associated. In fact one may, as the notation suggests, take the $r$-symbols as the first $r$ of the $s$-symbols. Let $u$ and $v$ be nonnegative integers $\leq s$ such that $u \equiv v(\bmod 2)$, that is, such that $u+v$ is even. A $(u, v)$-diagram is a matching of the $u+v$ symbols $y_{1}, \ldots, y_{u}, x_{1}, \ldots, x_{v}$ in pairs. Such a diagram is depicted as a graph whose vertices are arranged in two rows. The top row consists of $u$ vertices representing, from left to right, the $u$ covector symbols $y_{1}, \ldots, y_{u}$ and the bottom row consists of $v$ vertices representing the $v$ vector symbols $x_{1}, \ldots, x_{v}$. An empty row is represented by $\emptyset$. Two vertices are joined if the corresponding symbols are matched. So for example

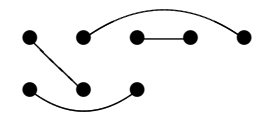

and

$\emptyset$

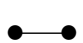

\footnotetext{
${ }^{5}$ elements of $V$ are called vectors and elements of $V^{*}$ are called covectors.
} 
are a $(5,3)$ and a $(0,2)$-diagram.

If an edge $e$ in a $(u, v)$-diagram is horizontal, then its left endpoint is called its initial point and its right endpoint is called its terminal point. If $e$ is not horizontal, then its endpoint in the top row is called its initial point and its endpoint in the bottom row is called its terminal point.

Let $t \in k$. We now define the $k$-algebra $\mathfrak{B}_{\leq s}(t)$. It has all $(u, v)$-diagrams, $0 \leq u, v \leq s, u \equiv v(\bmod 2)$, as a $k$-basis and its dimension is $\sum_{u=1}^{s} \sum_{v=1}^{s} N_{u v}$, where

$$
N_{u v}= \begin{cases}(u+v-1)(u+v-3) \cdots 3 \cdot 1=\frac{(u+v) !}{2^{(u+v) / 2}((u+v) / 2) !} & \text { if } u \equiv v(\bmod 2), \\ 0 & \text { otherwise. }\end{cases}
$$

The multiplication of diagrams is defined as follows. Let $D_{u v}$ be a $(u, v)$ diagram and let $D_{u^{\prime} v^{\prime}}$ be a $\left(u^{\prime}, v^{\prime}\right)$-diagram. Then we define

$$
D_{u v} D_{u^{\prime} v^{\prime}}= \begin{cases}\operatorname{sgn}\left(D_{u v}, D_{u^{\prime} v^{\prime}}\right) t^{\gamma(U)} D_{u v^{\prime}} & \text { if } v=u^{\prime}, \\ 0 & \text { if } v \neq u^{\prime} .\end{cases}
$$

Here, as in [3] and [13], $U$ is the graph which is obtained by putting $D_{u v}$ on top of $D_{u^{\prime} v^{\prime}}$ and identifying the vertices of the bottom row of $D_{u v}$ with those of the top row of $D_{u^{\prime} v^{\prime}}, \gamma(U)$ is the number of cycles in $U$ and $D_{u v^{\prime}}$ is the $\left(u, v^{\prime}\right)$-diagram obtained from $U$ by taking the vertices from top and bottom row in $U$ and matching two vertices if there is a path between them in $U$.

Our definition of the $\operatorname{sign} \operatorname{sgn}\left(D_{u v}, D_{u^{\prime} v^{\prime}}\right)$ of $D_{u v}$ over $D_{u^{\prime} v^{\prime}}$ does not agree with [13, p 411]. The graph $U$ consists of $\left(u+v^{\prime}\right) / 2$ paths and $\gamma(U)$ cycles that are of even length and consist entirely of edges in the middle row of $U$. A path in $U$ either consists of one horizontal edge in the top or bottom row of $U$ or it has precisely two non-horizontal edges and all its horizontal edges are in the middle row of $U$. A path of even length has one endpoint in the top row of $U$ and the other endpoint in the bottom row of $U$. A path of odd length has its endpoints both in the top row of $U$ or both in the bottom row of $U$.

Every path $P$ has a "natural" orientation. If $P$ has even length, then the endpoint in the top row is the initial point and the endpoint in the bottom row is the terminal point. If $P$ has odd length, then the leftmost endpoint of $P$ is the initial point and the other endpoint is the terminal point. For a path $P$ or an oriented cycle $P$ we denote the number of edges of $P$ in the middle row of $U$ that are traversed from left to right by $p_{l r}$ and the number of edges of $P$ in the middle row of $U$ that are traversed from right to left by $p_{r l}$. For a cycle or a path $P$ we define

$$
\operatorname{sgn}(P)= \begin{cases}(-1)^{\left|p_{l r}-p_{r l}\right| / 2} & \text { if } p_{l r}+p_{r l} \text { is even, } \\ (-1)^{\left|p_{l r}-p_{r l}-1\right| / 2} & \text { if } p_{l r}+p_{r l} \text { is odd. }\end{cases}
$$

Note that for a cycle this does not depend on the orientation. We now define $\operatorname{sgn}\left(D_{u v}, D_{u^{\prime} v^{\prime}}\right)$ as the product of the signs of all paths and cycles in $U$.

Below we will define a natural representation of $\mathfrak{B}_{\leq s}(n)$ which is the motivation for the definition of the multiplication. We will see later that the multiplication of $\mathfrak{B}_{\leq s}(t)$ is associative. 
With each $(u, v)$-diagram $D$ we can associate a $(u+v)$-multilinear function $F(D)$ on $\bigoplus^{u} V^{*} \oplus \bigoplus^{v} V$ as follows. First we observe that the form $\langle$, defines an isomorphism $V \cong V^{*}$, unique up to sign, and therefore a unique symplectic form on $V^{*}$ which we also denote by $\langle$,$\rangle . Furthermore we put$ $\langle y, x\rangle=y(x)=-\langle x, y\rangle$ for $y \in V^{*}$ and $x \in V$. If $e$ is an edge in $D$ whose initial point has label $z_{1}$ and whose terminal point has label $z_{2}$, then we put $\langle e\rangle=\left\langle z_{1}, z_{2}\right\rangle$. Now we define

$$
F(D)=\prod_{e \in D}\langle e\rangle .
$$

For example, for the first diagram $D$ in (6) we have

$$
F(D)=\left\langle y_{1}, x_{2}\right\rangle\left\langle y_{2}, y_{5}\right\rangle\left\langle y_{3}, y_{4}\right\rangle\left\langle x_{1}, x_{3}\right\rangle .
$$

Using the $k$-vector space isomorphisms

$$
\operatorname{End}_{k}\left(T^{\leq s}(V)\right) \cong \bigoplus_{0 \leq u, v \leq s} \operatorname{Hom}_{k}\left(\otimes^{v} V, \otimes^{u} V\right)
$$

and

$$
\operatorname{Hom}_{k}\left(\otimes^{v} V, \otimes^{u} V\right) \cong\left(\otimes^{u} V\right) \otimes\left(\otimes^{v} V\right)^{*} \cong\left(\left(\otimes^{u} V^{*}\right) \otimes\left(\otimes^{v} V\right)\right)^{*},
$$

we can now associate to each $(u, v)$-diagram $D$ an endomorphism $E(D)$ of $T^{\leq s}(V)$ as follows. We take $E(D)$ to be the endomorphism of $T^{\leq s}(V)$ corresponding to $F(D) \in\left(\left(\otimes^{u} V^{*}\right) \otimes\left(\otimes^{v} V\right)\right)^{*}$. Let $\left(e_{1}, \ldots, e_{n}\right)$ be the standard basis of $V=k^{n}$. Then we have a dual basis $\left(e_{1}^{*}, \ldots, e_{n}^{*}\right)$ and also bases of the vector spaces $\otimes^{r} V$. The matrix $M(D)$ of $E(D) \in \operatorname{Hom}_{k}\left(\otimes^{v} V, \otimes^{u} V\right)$ with respect to these bases is then given by

$$
M(D)_{\left(i_{1}, \ldots, i_{u}\right),\left(j_{1}, \ldots, j_{v}\right)}=F(D)\left(e_{i_{1}}^{*}, \ldots, e_{i_{u}}^{*}, e_{j_{1}}, \ldots, e_{j_{v}}\right) .
$$

The linear map $E: \mathfrak{B}_{\leq s}(n) \rightarrow \operatorname{End}_{k}\left(T^{\leq s}(V)\right)$ given by the assignment $D \mapsto$ $E(D)$ is a homomorphism of algebras. We indicate a proof.

The isomorphism $\varphi: V^{*} \rightarrow V$ with $\langle\varphi(y), x\rangle=y(x)$ for all $x \in V$ and $y \in V^{*}$ maps $e_{i}^{*}$ to $\epsilon_{i^{\prime}} e_{i^{\prime}}$. So we have

$$
\left\langle e_{i}^{*}, e_{j}^{*}\right\rangle=\left\langle e_{i}, e_{j}\right\rangle=\epsilon_{i} \delta_{i j^{\prime}} .
$$

Now one easily checks that

$$
\sum_{l_{2}, \ldots, l_{p}=1}^{n}\left\langle e_{l_{1}}, e_{l_{2}}\right\rangle\left\langle e_{l_{2}}, e_{l_{3}}\right\rangle \cdots\left\langle e_{l_{p}}, e_{l_{p+1}}\right\rangle= \begin{cases}(-1)^{p / 2}\left\langle e_{l_{1}}^{*}, e_{l_{p+1}}\right\rangle & \text { if } p \text { is even } \\ (-1)^{(p-1) / 2}\left\langle e_{l_{1}}, e_{l_{p+1}}\right\rangle & \text { if } p \text { is odd }\end{cases}
$$

and that for $p$ even

$$
\sum_{l_{1}, \ldots, l_{p}=1}^{n}\left\langle e_{l_{1}}, e_{l_{2}}\right\rangle\left\langle e_{l_{2}}, e_{l_{3}}\right\rangle \cdots\left\langle e_{l_{p-1}}, e_{l_{p}}\right\rangle\left\langle e_{l_{p}}, e_{l_{1}}\right\rangle=(-1)^{p / 2} \cdot n .
$$

If we evaluate an entry of the product $M\left(D_{u v}\right) M\left(D_{u^{\prime} v^{\prime}}\right), v=u^{\prime}$, then this entry will be a sum over $v$ indices corresponding to the vertices in the middle row of $U$. Here we use (7) and (10). Now we can distribute these $v$ sums over the paths and cycles in $U$. Each path or cycle gets the sums corresponding to the vertices 
of the middle row of $U$ that it contains. Then we obtain a product of sums of the above two types where we have to swap the arguments of certain scalar products $\left\langle e_{l_{i+1}}, e_{l_{i+2}}\right\rangle$ according to position of the corresponding vertices in the middle row of $U$. Here an extra sign $(-1)^{p_{l r}}$ comes in. Now it follows that the entry of $M\left(D_{u v}\right) M\left(D_{u^{\prime} v^{\prime}}\right)$ is equal to the corresponding entry of $M\left(D_{u v} D_{u^{\prime} v^{\prime}}\right)$.

We now define some special elements of $\mathfrak{B}_{\leq s}(t)$.

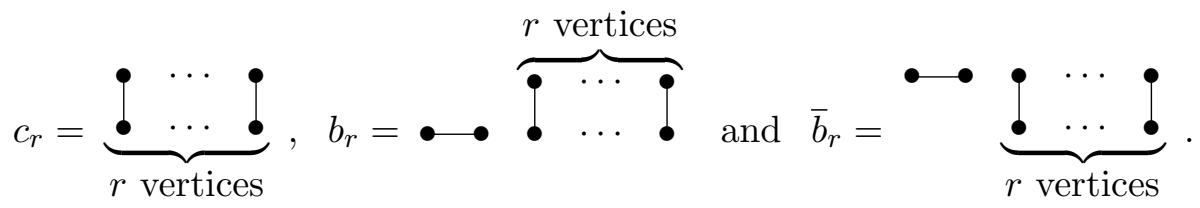

Here $c_{r}$ is defined for $0 \leq r \leq s$ and $b_{r}$ and $\bar{b}_{r}$ are defined for $0 \leq r \leq s-2$. The unit element of $\mathfrak{B}_{\leq s}(t)$ is $\sum_{r=0}^{s} c_{r}$. The subspace $c_{r} \mathfrak{B}_{\leq s}(t) c_{r}$ is the span of all $(r, r)$-diagrams and is closed under multiplication. It has $c_{r}$ as a unit element and as an algebra it is isomorphic to the symplectic Brauer algebra $\mathfrak{B}_{r}(t)$ which was introduced for $t=n$ in [3]. We identify $\mathfrak{B}_{r}(t)$ with this subspace of $\mathfrak{B}_{\leq s}(t)$. Then $\bigoplus_{r=0}^{s} \mathfrak{B}_{r}(t)$ is identified with a subalgebra with unit of $\mathfrak{B}_{\leq s}(t)$. We obtain a natural embedding $k\left\langle\operatorname{Sym}_{r}\right\rangle \subseteq \mathfrak{B}_{r}(t)$ by assigning to each $\pi \in \operatorname{Sym}_{r}$ the $(r, r)$ diagram in which $x_{i}$ is matched with $y_{\pi_{i}}$. The action of $\operatorname{Sym}_{r}$ on $\otimes^{r} V$ that it inherits from $\mathfrak{B}_{r}(n)$ is the same as the action mentioned in Section 1.

The algebra $\mathfrak{B}_{\leq s}(t)$ is generated by the elements $b_{r}, \bar{b}_{r}, 0 \leq r \leq s-2, c_{0}, c_{1}$ and the elements of each $\mathfrak{B}_{r}(t), 2 \leq r \leq s$, that correspond to the elementary transpositions in $\mathrm{Sym}_{r}$.

In the theorem below we will consider the representation of $\mathfrak{B}_{\leq s}(n)$ on $T^{\leq s}(V)$ as given by the homomorphism $E$ defined above. First we make a preliminary observation. Let $B \in \operatorname{Hom}_{k}(V \otimes V, k) \subseteq \operatorname{End}_{k}\left(T^{\leq s}(V)\right.$ be the endomorphism that maps $x_{1} \otimes x_{2}$ to $\left\langle x_{1}, x_{2}\right\rangle$ and let $\bar{B} \in \operatorname{Hom}_{k}(k, V \otimes V) \subseteq \operatorname{End}_{k}\left(T^{\leq s}(V)\right.$ be the endomorphism that maps 1 to $\sum_{i=1}^{n} \epsilon_{i} e_{i} \otimes e_{i^{\prime}}$. Then we have

$$
E\left(b_{r}\right)=B \otimes \operatorname{id}_{\otimes^{r} V} \quad \text { and } \quad E\left(\bar{b}_{r}\right)=\bar{B} \otimes \operatorname{id}_{\otimes^{r} V} .
$$

Note furthermore that $E\left(c_{r}\right)$ is just the projection of $T^{\leq s}(V)$ onto $\otimes^{r} V$.

Theorem 3. The following holds.

(i) $\operatorname{End}_{\mathrm{Sp}_{n}}\left(T^{\leq s}(V)\right)$ coincides with the image of $\mathfrak{B}_{\leq s}(n)$ in $\operatorname{End}_{k}\left(T^{\leq s}(V)\right)$.

(ii) $\operatorname{End}_{\mathfrak{B}_{\leq s}(n)}\left(T^{\leq s}(V)\right)$ is the enveloping algebra of $\operatorname{Sp}_{n}$ in $\operatorname{End}_{k}\left(T^{\leq s}(V)\right)$.

(iii) If $m \geq s$, then the homomorphism $\mathfrak{B}_{\leq s}(n) \rightarrow \operatorname{End}_{k}(T \leq s(V))$ is injective.

(iv) If $m<s$, then the homomorphism $\mathfrak{B}_{s}(n) \rightarrow \operatorname{End}_{k}\left(\otimes^{s} V\right)$ is not injective.

Proof. (i). We follow Brauer's method as adjusted to our situation by Weyl, see $\left[20, \mathrm{~V} .2, \mathrm{p}\right.$ 141-142]. There is a natural action of $\operatorname{Sp}_{n}$ on $\operatorname{End}_{k}\left(T^{\leq s}(V)\right)$ and it is clear that $\operatorname{End}_{\mathrm{Sp}_{n}}\left(T^{\leq s}(V)\right)$ consists of the $\mathrm{Sp}_{n}$-invariant elements of $\operatorname{End}_{k}\left(T^{\leq s}(V)\right)$. The vector spaces in (8) and (9) all have a natural $\mathrm{Sp}_{n}$-action and the isomorphisms there are $\mathrm{Sp}_{n}$-equivariant. It therefore suffices to show that for $u, v \in\{1, \ldots, s\},\left(\left(\otimes^{u} V^{*}\right) \otimes\left(\otimes^{v} V\right)\right)^{* \mathrm{Sp}_{n}}$ is spanned by the multilinear functions $F(D)$, where $D$ is a $(u, v)$-diagram and $F$ is given by (7).

Let $u, v \in\{1, \ldots, s\}$ and put $w=u+v$. We will identify $V^{*}$ with $V$ by means of the isomorphism $\varphi: V^{*} \stackrel{\sim}{\rightarrow} V$. This means that the $y_{i}$ are now vector 
variables. We put $z_{i}=y_{i}$ for $i \in\{1, \ldots, u\}$ and $z_{i}=x_{i-u}$ for $i \in\{u+1, \ldots, w\}$. Since $k$ is infinite, $k\left[\oplus^{w} V\right]$ can be identified with the polynomial ring in the components of the $z_{i}$. If $f \in k\left[\oplus^{w} V\right]$ is $\operatorname{Sp}_{n}$-invariant, then it is also $\operatorname{Sp}_{n}(K)$ invariant as an element of $K \otimes_{k} k\left[\oplus^{w} V\right]$, since $\operatorname{Sp}_{n}$ is dense in $\operatorname{Sp}_{n}(K)$. But then $f$ is a formal invariant in the definition of $[6, \S 2]$, see e.g. [14, Remark I.2.8]. We can now apply the first fundamental theorem of invariant theory for the symplectic group, [6, Thm. 6.6]. This gives us that $k\left[\oplus^{w} V\right]^{\mathrm{Sp}_{n}}$ is generated as a $k$-algebra by the scalar products $\left\langle z_{i}, z_{j}\right\rangle, 1 \leq i<j \leq w$. It follows immediately that $\left(\otimes^{w} V\right)^{* \mathrm{Sp}_{n}}$ is spanned by the monomials in the $\left\langle z_{i}, z_{j}\right\rangle, 1 \leq i<j \leq w$, in which each $z_{i}$ occurs exactly once. These monomials are precisely the $F(D)$, where $D$ is a $(u, v)$-diagram.

(ii). We have $\mathfrak{S} \subseteq \mathfrak{B}_{\leq s}(n)$, where $\mathfrak{S}$ is as in Proposition 1 (iv). So, as in the proof of that result, we have that $\operatorname{End}_{\mathfrak{B}_{<s}(n)}\left(T^{\leq s}(V)\right)$ consists of $(s+1)$-tuples of bisymmetric substitutions. Using (12) one easily checks that the condition of commuting with $E\left(b_{r}\right)$ is given by the equation (4) and that (5) gives the condition of commuting with $E\left(\bar{b}_{r}\right)$. By Theorem 1(iii) these are precisely the equations that define $\mathcal{E}^{\leq s}\left(\mathrm{Sp}_{n}\right)$.

The arguments in the proofs of (iii) and (iv) below are very similar to those for the orthogonal group in [20, p 149]. Recall that the Pfaffian of an alternating $2 r \times 2 r$-matrix A is defined by

$$
\operatorname{Pf}(A)=\sum_{\pi} \operatorname{sgn}(\pi) \prod_{i=1}^{r} a_{\pi_{2 i-1}, \pi_{2 i}},
$$

where the sum is over all permutations $\pi$ of $\{1,2, \ldots, 2 r\}$ with $\pi_{1}<\pi_{3}<\cdots<$ $\pi_{2 r-1}$ and $\pi_{2 i-1}<\pi_{2 i}$ for all $i \in\{1, \ldots, r\}$.

(iii). By the isomorphisms (8) and (9) it is sufficient to show that for each $u, v \in 1, \ldots, s$ with $u \equiv v(\bmod 2)$, the multilinear functions $F(D), D$ a $(u, v)$ diagram, are linearly independent. We use the identification $V^{*} \cong V$ and the notation of (i). As we have seen in (i) the $F(D)$ are the monomials in the $\left\langle z_{i}, z_{j}\right\rangle$, $1 \leq i<j \leq w$ in which each $z_{i}$ occurs exactly once. The second fundamental theorem for the symplectic group, [6, Thm. 6.7], says that the ideal of relations between the $\left\langle z_{i}, z_{j}\right\rangle, 1 \leq i<j \leq w$ is generated by the Pfaffians of the principal submatrices of size $n+1$ of the $w \times w$ alternating matrix $\left\langle z_{i}, z_{j}\right\rangle_{1 \leq i, j \leq w}{ }^{6}$ Since, by assumption, $w \leq 2 s \leq n$, there are no such submatrices, that is, the $\left\langle z_{i}, z_{j}\right\rangle$, $1 \leq i<j \leq w$ are algebraically independent. This means that the monomials in the $\left\langle z_{i}, z_{j}\right\rangle, 1 \leq i<j \leq w$ are linearly independent.

(iv). From (13) it is clear that $\operatorname{Pf}(A)$ is a signed sum of distinct monomials in the $a_{i j}, 1 \leq i<j \leq 2 r$, in which each number $i \in\{1,2, \ldots, 2 r\}$ occurs precisely once either as row or as column index. Since $n<2 s$, we must have that the alternating $2 s$-multilinear map $\operatorname{Pf}\left(\left\langle z_{i}, z_{j}\right\rangle_{1 \leq i, j \leq 2 s}\right)$ on $\oplus^{2 s} V$ is zero. This gives us a nontrivial dependence relation between the multilinear functions $F(D), D$ an $(s, s)$-diagram.

Corollary. The algebra $\mathfrak{B}_{\leq s}(t)$ is associative for any $t \in k$.

\footnotetext{
${ }^{6} \mathrm{~A}$ principal submatrix is obtained by choosing rows and columns from the same index set.
} 
Proof. First we observe that we can define the Brauer algebra over any commutative ring $R$ and then we have $\mathfrak{B}_{\leq s}\left(R, t_{0}\right) \cong R \otimes_{\mathbb{Z}[t]} \mathfrak{B}_{\leq s}(\mathbb{Z}[t], t)$ for all $t_{0} \in R$, where we now consider $t$ as an indeterminate and the homomorphism $\mathbb{Z}[t] \rightarrow R$ is given by $t \mapsto t_{0}$. So it suffices to show that $\mathfrak{B}_{\leq s}(\mathbb{Z}[t], t)$ is associative. If $D, D^{\prime}, D^{\prime \prime} \in \mathfrak{B}_{\leq s}(\mathbb{Z}[t], t)$, then the coefficients of $\left(D D^{\prime}\right) D^{\prime \prime}-D\left(D^{\prime} D^{\prime \prime}\right)$ with respect to the diagram basis are polynomials in $t$. By Theorem 3(iii) applied with $k=\mathbb{Q}$, these polynomials vanish whenever we specialise $t$ to an even integer $\geq 2 s$. It follows that these polynomials are identically zero.

Remarks 3. 1. Theorem 3(iii) was proved in another way in [10, Thm. 3.4]. 2. One can also derive the double centraliser theorem for $\mathrm{Sp}_{n}$ acting on $\otimes^{s} V$ as obtained in [10, Prop. 1.3 and Thm 1.4] by our method. Simply combine Remark 2.2 and the arguments of the proof of Theorem 3.

3 . Drop the assumption that $n$ is even and assume that the characteristic of $k$ is not 2. Orthogonal versions $\mathfrak{A}_{<s}(t)$ and $\mathfrak{A}_{s}(t)$ of $\mathfrak{B}_{<s}(t)$ and $\mathfrak{B}_{s}(t)$ can also be defined. One just has to omit the sign in the definition of the multiplication. The algebra $\mathfrak{A}_{s}(t)$ coincides with the orthogonal Brauer algebra defined in [13]. Furthermore the algebras $\mathfrak{A}_{\leq s}(n)$ and $\mathfrak{A}_{s}(n)$ have natural representations in $T^{\leq s}(V)$ and $\otimes^{s} V$.

Let $I$ be the orthogonal ideal. In characteristic zero homogeneous generators for $I_{h o m}$ are given in [9, Thm. 10.5a)] and generators for $I$ with property (1) are given in [20, Thm. 5.2.C]. The Corollary to Proposition 1 and the proof of Theorem 3 show that, to prove the double centraliser theorem over $k$ for the orthogonal group acting on $\otimes^{s} V$ and $T^{\leq s}(V)$, it is enough to show that these elements are also generators of $I_{h o m}$ and generators for $I$ with property (1) over $k$.

4. Assume that $k=\mathbb{C}$. Then it can be shown by easy invariant theoretic arguments as in the proof of Theorem 3(iii) and (iv) that the natural representation $\mathfrak{A}_{s}(n) \rightarrow \operatorname{End}_{k}\left(\otimes^{s} V\right)$ is injective if $n \geq 2 s$ and not injective if $n<s$; see [20, p 149]. So, contrary to the symplectic case, these arguments do not give a complete criterion for faithfulness. In [4] it is proved that the natural representation of $\mathfrak{A}_{s}(n)$ is faithful if and only if $n \geq s$.

5. From $[17$, Thm.'s $2.11,4.6,4.3]$ it follows that $\mathfrak{A}_{s}(t)$ has the well-known presentation given in e.g. [10, p 3]. From this one easily deduces a presentation for $\mathfrak{B}_{s}(t)$ and an isomorphism $\mathfrak{B}_{s}(t) \cong \mathfrak{A}_{s}(-t)$. This is in accordance with the results in [13] and [19].

Acknowledgement. I would like to thank S. Oehms for his comments on a preliminary version of this paper and A. M. Cohen for bringing [17] to my attention. This research was funded by the EPSRC Grant EP/C542150/1.

\section{REFERENCES}

[1] A. Berele, Construction of Sp-modules by tableaux, Linear and Multilinear Algebra 19 (1986), no. 4, 299-307.

[2] N. Bourbaki, Commutative algebra, Chapters 1-7, Translated from the French, Reprint of the 1972 edition, Springer, Berlin, 1989.

[3] R. Brauer On algebras which are connected with the semisimple continuous groups, Ann. of Math. (2) 38 (1937), no. 4, 857-872.

[4] W. P. Brown, The semisimplicity of $\omega_{f}^{n}$, Ann. of Math. (2) 63 (1956), 324-335. 
[5] C. De Concini, Symplectic standard tableaux, Adv. in Math. 34 (1979), no. 1, 1-27.

[6] C. De Concini, C. Procesi, A characteristic free approach to invariant theory, Advances in Math. 21 (1976), no. 3, 330-354.

[7] S. Donkin, Good filtrations of rational modules for reductive groups in The Arcata Conference on Representations of Finite Groups (Arcata, Calif., 1986), Proc. Sympos. Pure Math. 47:1, Amer. Math. Soc., Providence, RI, 1987, 69-80.

[8] S. Donkin, Representations of symplectic groups and the symplectic tableaux of $R$. $C$. King, Linear and Multilinear Algebra 29 (1991), no. 2, 113-124.

[9] S. Doty, Polynomial representations, algebraic monoids, and Schur algebras of classical type, J. Pure Appl. Algebra 123 (1998), no. 1-3, 165-199.

[10] R. Dipper, S. Doty, J. Hu, Brauer algbras, symplectic Schur algebras and Schur-Weyl duality, to appear in Trans. Amer. Math. Soc.

[11] D. Eisenbud, Commutative Algebra with a View Toward Algebraic Geometry, Graduate Texts in Math., vol. 150, Springer, New York, 1995.

[12] J. A. Green, Polynomial representations of $\mathrm{GL}_{n}$, Lecture Notes in Mathematics, 830, Springer-Verlag, Berlin-New York, 1980.

[13] P. Hanlon, D. Wales, On the decomposition of Brauer's centralizer algebras, J. Algebra 121 (1989), no. 2, 409-445.

[14] J. C. Jantzen, Representations of algebraic groups, Pure and Applied Math., vol. 131. Academic Press, Boston, 1987.

[15] S. Oehms, Centralizer coalgebras, FRT-construction, and symplectic monoids, J. Algebra 244 (2001), no. 1, 19-44.

[16] R. C. King, Weight multiplicities for the classical groups, Group theoretical methods in physics (Fourth Internat. Colloq., Nijmegen, 1975), Lecture Notes in Phys., Vol. 50, Springer, Berlin, 1976, 490-499.

[17] H. R. Morton, A. J. Wasserman, A basis for the Birman-Wenzl Algebra, preprint, 1989, http://citeseer.ist.psu.edu/313132.html.

[18] R. H. Tange, Infinitesimal invariants in a function algebra, to appear in Canad. J. Math.

[19] H. Wenzl, On the structure of Brauer's centralizer algebras, Ann. of Math. (2) 128 (1988), no. $1,173-193$.

[20] H. Weyl, The classical groups, Their invariants and representations, second edition, Princeton University Press, 1946.

Fakultät Für Mathematik, Ruhr-Universität Bochum, Universitätsstrasse 150, D-44780 Bochum, Germany

E-mail address: rudolf.tange@rub.de 